\title{
PRESENÇA49 DE CÁDMIO EM MOSTOS E VINHOS
}

\author{
CADMIUM IN MUSTS AND WINES
}

\author{
Denise D'Agostini ${ }^{1}$ Carlos Eugênio Daudt ${ }^{2}$
}

\section{RESUMO}

Cádmio foi determinado em mostos e vinhos de duas cultivares de uvas vim/eras (Cabernet Sauvignon e Saint Emilion). Amostras/oram retiradas periodicamente durante a fermentação alcoólica e também do bagaço da $\mathrm{cv}$. Cabernet Sauvignon, das borras e das sementes resultantes das trasfegas $e$ do produto final. As fermentações de cada cultivar sofreram adição de quantidades conhecidas de cádmio ( $5 \mu \mathrm{g} / \mathrm{l}$ e $5 \mathrm{mg} / \mathrm{l})$ afim de verificar o seu comportamento em diferentes concentrações. As determinações de cádmio foram realizadas através da espectrometria de absorção atómica com utilização de forno de grafite. Os valores máximos encontrados, expressos em $\mu \mathrm{g} / 1$ foram, respectivamente: vinhos controle, 0,88: vinhos adicionados de baixos teores de cádmio $(5 \mu \mathrm{g} / \mathrm{l}), 4,17: e$, vinhos adicionados de altos teores de cádmio (5mg/l), 280: as percentagens retidas nos vinhos com relação ao mosto original oscilaram entre $32,5 \%$ e $60,1 \%$. Os valores encontrados para cádmio podem ser considerados baixos quando comparados com os relatados na literatura.

Palavras-chave: metal, suco de uva, fermentação

\section{SUMMARY}

Cadmium v/as analyzed in musts anã wines oftwo grapes varieties, Vitis vinifera Cabernet Sauvignon and Saint Emilion. Samples were taken during the alcohoolicfermentation as well as from the cap, seeds, lees and wines. Different quantities $(5 \mu \mathrm{g}$ and $5 \mathrm{mg} / \mathrm{l})$ of cadmiun were added prior to start the fermentations. Cadmium was analysed by atomic absorption spectrometry using a graphite oven. The maximum values found, in $\mu \mathrm{g} / \mathrm{l}$, were respectively: wines contrai, 0.88 : wines from musts with low Cdaddition $(5 \mu \mathrm{g} / \mathrm{l}), 4.17$ and wines from musts with high Cd addition $(5 \mathrm{mg} / \mathrm{l}), 280$. The cadmium contem of the wines ranged from $32.5 \%$ and $60.1 \%$ of the cadmium originalty present in the musts. However, these amounts were considered low.

Key words: metal, grapejuice.fermentation.

\section{INTRODUÇÃO}

Cádmio é um elemento presente em baixas concentrações na natureza apresentando características tóxicas e carcinogênicas (LEMEN $\boldsymbol{e t}$ al., 1975). As videiras situam-se entre as plantas cultivadas mais sensíveis a este metal pesado (ENKELMANN, 1987) manifestando seus efeitos tóxicos quando cultivadas em solos ácidos (DELAS, 1984). Os principais efeitos tóxicos do cádmio sobre as videiras são: inibição do crescimento dos brotos, diminuição da área foliar, clorose, necrose, deformações foliares, redução da produção de matéria seca, redução dos níveis de clorofila e inibição do transporte de ferro das raízes até as folhas causando deficiência deste mineral (RUPP $\boldsymbol{e t}$ al., 1985).

A presença de cádmio em vinhos pode ser devida a uma fração primária ou natural chegando até os mesmos por via das raízes, das folhas e das uvas. A fração secundária aparece nos vinhos através de adulterações ou contaminações durante o seu processamento (ESCHNAUER, 1982).

\footnotetext{
${ }^{1}$ Farmacêutico-bioquímico, técnico de laboratório, aluna do Mestrado em Ciência e Tecnologia dos Alimentos, Vinhos. Parte da dissertação.

${ }^{2}$ Engenheiro Agrônomo, PhD., Professor Titular do Departamento de Tecnologia e Ciência dos Alimento, Centro de Ciências Rurais Universidade Federal de Santa Maria, 97119-900, Santa Maria - RS, Brasil. Autor para correspondência.
} 
Existe um crescente interesse de organizações como a FAO (Food Agriculture Organization) e a WHO (Worid Health Organization) pela determinação de contaminantes químicos em alimentos (ROSENEGGER, 1992). No Brasil, embora existam vários estudos de minerais em mostos e vinhos (DAUDT \& GARCIA, 1987; DAUDT $\boldsymbol{e}$ t al., 1987; DAUDT \& DAL PI VA, 1989 e DAUDT $\boldsymbol{e t}$ al., 1992), ainda há poucas publicações de cádmio na área de viticultura e enologia. Os objetivos deste trabalho foram analisar cádmio em mostos, vinhos, borras e sementes.

\section{MATERIAIS E MÉTODOS}

Foram utilizadas duas cultivares de Vitis vinífera: Cabemet Sauvignon e Saint Emilion. A cultivar tinta foi proveniente de Santana do Livramento (Almadén Vinhos Finos Ltda.) e de um vinhedo particular localizado no município de São Sepé, RS. A cultivar branca foi proveniente de Santana do Livramento (Almadén Vinhos Finos Ltda.). Por ocasião da colheita as uvas apresentaram a composição constante na Tabela 1 .

Tabela 1 - Caracterização e origem das uvas usadas neste experimento.

\begin{tabular}{llccc}
\hline Cultivar & $\begin{array}{l}\text { Município } \\
\text { de origem }\end{array}$ & ${ }^{\circ}$ Brix & $\mathrm{pH}$ & $\begin{array}{c}\text { Ac. Total } \\
(\mathrm{gH} 2 \mathrm{Ta} / 100 \mathrm{ml})\end{array}$ \\
\hline Cabernet Sauvignon & São Sepé & 20 & 3,20 & 0,65 \\
Cabernet Sauvignon & Livramento & 24 & 3,83 & 0,43 \\
Saint Emilion & Livramento & 23 & 3,53 & 0,51 \\
\hline
\end{tabular}

*gH2Ta = gramas de ácido tartático.

Após o esmagamento e desengaçamento manual o mosto de cada cultivar foi dividido em 2 lotes de 5 litros cada. As cultivares oriundas de Santana do Livramento além do tratamento controle (sem adição de cádmio) tiveram um tratamento com adição de $5 \mu \mathrm{g}$ de $\mathrm{Cd} / \mathrm{l}$; por outro lado, a cultivar oriunda de São Sepé além do tratamento controle (sem adição de cádmio) teve um tratamento com $5 \mathrm{mgCd} / 1$. Esta diferença entre os dois tratamentos foi consequência do desconhecimento total sobre as quantidades de cádmio existentes em uvas do Rio Grande do Sul, sendo, portanto, necessário adicionar cádmio numa quantidade variável. Cádmio foi adicionado na forma de $\mathrm{CdC} 12$ (cloreto de cádmio, ampola Merck que dissolvida em 1 litro de água forma solução de $1 \mathrm{~g} / \mathrm{l}$ ). Logo a seguir os mostos sofreram a adição de $50 \mathrm{mg} / \mathrm{l}$ de $\mathrm{S} 02$ e foram inoculados com Saccharomyces cerevisiae Montrachet. Os mostos brancos fermentaram a 18 "C e os tintos entre 25 "C e $28{ }^{\circ} \mathrm{C}$. Nos tintos as películas foram retiradas ao redor de $5^{\circ}$ Brix e o término da fermentação $(0,2 \%$ açúcar $)$ foi determinado pela análise de açúcar redutor conforme o método descrito por AMERINE \& OUGH (1974). Após duas trasfegas e estabilização os vinhos foram engarrafados. Amostras do mosto foram retiradas após os tratamentos, periodicamente durante a fermentação, no final da mesma, após a refrigeração e um mês após o engarrafamento. Também foram coletadas amostras das películas e sementes da cv. tinta (chapéu e bagaço), das borras do final das fermentações e das borras depositadas após refrigeração e estabilização dos vinhos. Todos os lotes de fermentação foram realizados com duas repetições.

As amostras de mostos (colhidas em duplicata) foram evaporadas em banho-maria a uma temperatura de $55^{\circ} \mathrm{C}$ até concentração de $20 \%$ do volume original. Posteriormente estas amostras foram digeridas em bloco digestor a uma temperatura de $60^{\circ} \mathrm{C}$ até limpidez total do líquido. Finalmente, foi efetuada uma diluição com água destilada-deionizada até o volume original. As determinações de cádmio foram realizadas utilizando-se um espectrofotômetro de absorção atómica da marca PERKIN-ELMER2380 provido de forno de grafite HGA 300. Foi empregada uma lâmpada de cátodo oco de comprimento de onda $(\lambda)$ igual a $228,8 \mathrm{~nm}$. A temperatura de secagem da amostra foi de $130^{\circ} \mathrm{C}$, sendo que a temperatura de condicionamento foi de $300^{\circ} \mathrm{C}$ e a de atomização igual a $2.000^{\circ} \mathrm{C}$. Estas análises também foram sempre realizadas em duplicata. Para a análise estatística dos resultados foi realizado o delineamento experimental inteiramente casualizado. A fim de comparar as diferenças entre as médias aplicou-se o teste Duncan a $5 \%$ de signifícância conforme STEEL \& TORRIE (1980).

\section{RESULTADOS E DISCUSSÃO}

A Tabela 2 mostra o teor de cádmio em amostras obtidas durante as diferentes etapas do processo de vinificação nos diferentes tratamentos. Deve ser observado que a toxicidade do cádmio encontra-se associada à sua capacidade de substituir elementos essenciais como o cobre e o zinco, os quais 


\begin{tabular}{|c|c|c|c|c|c|c|}
\hline \multirow{2}{*}{ Elapas da vinificacio } & \multicolumn{2}{|c|}{$\mathrm{Cs}^{*}$} & \multicolumn{2}{|c|}{$\mathrm{Cs}^{*} *$} & \multicolumn{2}{|c|}{$\mathrm{SE}^{*}$} \\
\hline & $\begin{array}{l}\text { Controle } \\
\text { Hg/l }\end{array}$ & $\begin{array}{l}58 \mathrm{Cl} \\
\mathrm{Hg} /\end{array}$ & Controle & $\begin{array}{l}5 \mathrm{Cd} \\
\mathrm{mg} /\end{array}$ & $\begin{array}{l}\text { Controle } \\
\text { ugl }\end{array}$ & $\begin{array}{l}5 \mathrm{Cd} \\
\text { ugh }\end{array}$ \\
\hline $\begin{array}{l}\text { Inicio da fermentagio } \\
\text { Pim da fermentacdo } \\
\text { Após resfriamento } \\
\text { Apos engarrafamento }\end{array}$ & $\begin{array}{l}2,42 \mathrm{Ba} \\
1,45 \mathrm{Bb} \\
0,91 \mathrm{Bc} \\
0,88 \mathrm{Bc}\end{array}$ & $\begin{array}{l}6,48 \mathrm{Ba} \\
3,58 \mathrm{Ab} \\
3,86 \mathrm{Ac} \\
3,77 \mathrm{Ac}\end{array}$ & $\begin{array}{l}2,1,5 \mathrm{a} \\
1,14 \mathrm{~b} \\
0,69 \mathrm{c} \\
0,70 \mathrm{c}\end{array}$ & $\begin{array}{l}0,58 \mathrm{a} \\
0,35 \mathrm{~b} \\
0,31 \mathrm{c} \\
0,28 \mathrm{c}\end{array}$ & $\begin{array}{l}2,14 \mathrm{Ba} \\
1,09 \mathrm{Bb} \\
0,78 \mathrm{BC} \\
0,76 \mathrm{BC}\end{array}$ & $\begin{array}{l}7,23 \mathrm{pa} \\
5,83 \mathrm{Ab} \\
4,25 \mathrm{Ac} \\
4,17 \mathrm{Ac}\end{array}$ \\
\hline $\begin{array}{l}\text { e SE = Cabem } \\
S=C \text { abernet } S\end{array}$ & on $p$ & $\mathrm{E}_{1}$ & ove & dentro & $\begin{array}{l}\text { rzonts } \\
\text { ada cu }\end{array}$ & $\begin{array}{l}\text { nusct } \\
\text { ocal. } \\
\text { to. }\end{array}$ \\
\hline
\end{tabular}

hipótese, afirmando que a fonte de sulfeto é o ácido suifídrico produzido pelas leveduras durante a fermentação; constatou, também, que a eliminação de cádmio resultante da fermentação estava compreendida entre $34 \%$ e $79 \%$. O percentual de cádmio eliminado durante a fermentação (Tabela 2) foi de $40,1 \%$ e $13,9 \%$ para Cabemet Sauvignon de Santana do Livramento (para o controle e $5 \mu \mathrm{g} / 1$

funcionam como cofatores de enzimas vitais para as leveduras, tais como a superóxido dismutase (KOFOD et al., 1991). Provavelmente não foram utilizadas, neste experimento, concentrações de cádmio suficientemente tóxicas capazes de afetar a multiplicação e o crescimento das leveduras. Segundo ANGLE et al., (1992), a toxicidade do cádmio é influenciada pela concentração de nutrientes de cultura. Num meio rico em nutrientes como, por exemplo, o mosto de uva, as leveduras apresentam uma maior tolerância a este metal tóxico. Desta forma, acredita-se que as leveduras em questão foram capazes de suportar concentrações elevadas deste metal $(5 \mathrm{mg} / \mathrm{l})$ sem haver prejuízos nas fermentações. MOHR (1979), trabalhando com mostos da cv. Riesling, constatou que os efeitos do cádmio, retardando as fermentações, eram observados em concentrações entre 2,0 e 5,0mg/l. OLASÜPO et al., (1993), em estudo relacionado com a seleção de leveduras para a indústria de bebidas alcoólicas, encontraram cepas de Saccharomyces cerevisiae resistentes ao cádmio em concentrações de até $1686 \mathrm{mg} / \mathrm{l}$. Segundo WINGE $\boldsymbol{e} t$ al (1992) as leveduras apresentam um mecanismo de detoxificação de cádmio. Elas são capazes de sequestrar este metal intracelularmente sob a forma de cristais de sulfeto de cádmio, ficando este metal isolado, não afetando a atividade metabólica destes microorganismos.

Os resultados demonstraram em todos os casos uma diminuição nos teores de cádmio durante o processo de fermentação (Figuras 1 e 2). Isto provavelmente ocorreu, em parte, devido à precipitação de sais insolúveis de cádmio e, em parte, devido ao consumo deste elemento pelas leveduras. Segundo GAERTEL (1984), o cádmio é precipitado em sua maior parte durante a fermentação sob a forma de sulfeto de cádmio. MOHR (1979) concorda com esta adicionado), $47,1 \%$ e $38,7 \%$ para Cabemet Sauvignon de São Sepé (controle e $5 \mathrm{mg} / 1$ adicionado). 49,1\% e 19,4\% para Saint Emilion (controle e $5 \mu \mathrm{g} / 1$ adicionado respectivamente). Os fatores que determinam a formação de $\mathrm{H}_{2} \mathrm{~S}$ também determinam a precipitação de cádmio. ESCHENBRUCH (1974) afirma que certos íons metálicos estimulam a formação de $\mathrm{H}_{2} \mathrm{~S}$ pelas leveduras. Desta forma, se o cádmio fosse um provável estimulador poderia atuar em concentrações elevadas (como por exemplo $5 \mathrm{mg} / \mathrm{l}$ ), mas não em baixas concentrações (como por exemplo $5 \mu \mathrm{g} / \mathrm{l}$ ) o que concorda, parcial- mente, com os resultados encontrados. Assim, quando o cádmio se encontra presente no meio em altas concentrações, estimularia a produção de maiores quantidades de $\mathrm{H}_{2} \mathrm{~S}$, o que aumentaria o percentual de precipitações.

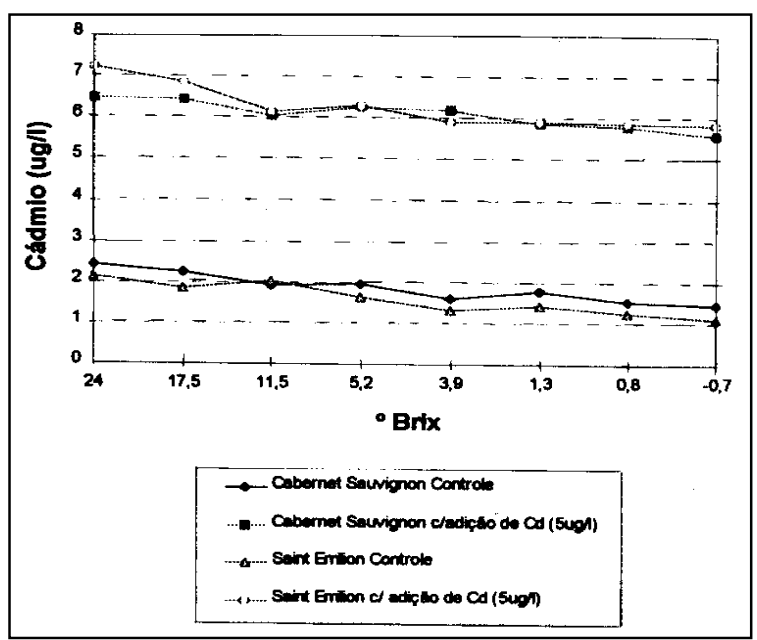

Figura 1 - Evolução dos teores de Cádmio $(\mu \mathrm{g} / \mathrm{l})$ durante as fermentações dos mostos das cvs Cabernet Sauvignon e Saint Emilion oriundas de Santana do Livramento com diferentes tratamentos. 


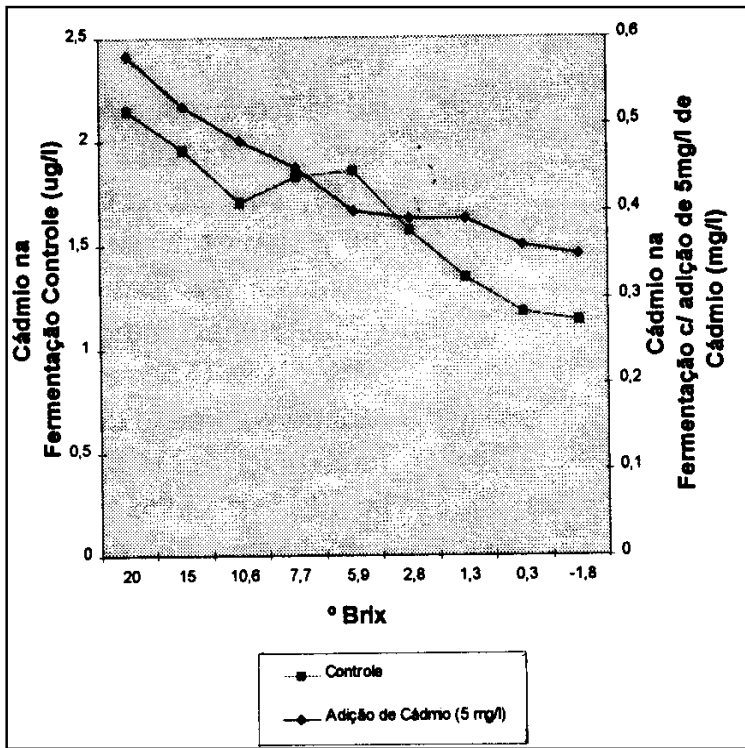

Figura 2 - Evolução dos teores de Cádmio durante a fermentação dos mostos da cv. Cabernet Sauvignon oriunda de São Sepé com os diferentes tratamentos.

Nos mostos adicionados com altos teores de cádmio $(5 \mathrm{mg} / \mathrm{l})$ ocorreu uma diminuição acentuada de cádmio logo no início da fermentação (Tabela 2). Neste primeiro momento, provavelmente, como o $\mathrm{H}_{2} \mathrm{~S}$ não se encontrava ainda presente no meio esta precipitação pode ter ocorrido devido à deposição de algum outro sal de cádmio como fosfato de cádmio que também apresenta baixa solubilidade (COTTON \& WILKINSON, 1978).

A aplicação de baixas temperaturas (resfriamento) também ocasionou uma diminuição significativa no teor de cádmio dos vinhos (Tabela 2). Isto era esperado, pois, diminuindo-se a temperatura de uma solução a solubilidade da maioria dos sais também diminui.

Em relação ao teor de cádmio presente no mosto original, o conteúdo final deste metal, nos vinhos engarrafados que não sofreram adição, esteve compreendido entre $32,5 \%$ e $36,4 \%$ do inicialmente presente. Por outro lado, nas fermentações em que cádmio foi adicionado, o percentual remanescente no vinho esteve entre $48,3 \%$ e $58,2 \%$ (Tabela 2).

O conteúdo final de cádmio dos vinhos
(Tabela 2), exceto daquele oriundo do mosto com altas concentrações deste metal adicionado $(5 \mathrm{mg} / \mathrm{l})$, sempre esteve abaixo do limite máximo estipulado pela O.I.V. (Offíce Intemational de laVigne et du Vin) que, de acordo com WENCKER et al. (1990) é de $10 \mu \mathrm{g} / 1$. Os valores encontrados nos vinhos coincidem com aqueles citados por diversos autores, entre 0,1 e 8,0 $\mu \mathrm{g} / \mathrm{l}$ (ESCHNAUER, 1982; URENAPOZO et al., 1987; ARCOS et al., 1993 e ANDREY et al., 1992).

As análises de cádmio nas películas e sementes (Tabela 3) da cv. Cabemet Sauvignon proveniente do município de Santana do Livramento e de São Sepé indicaram que somente uma pequena parte do cádmio foi depositado neste material, e que esta deposição ocorreu de maneira proporcional à quantidade depositada durante as fermentações (Tabela 2). Os valores encontrados para cádmio nas borras (exceto nos tratamentos onde adicionou-se $5 \mathrm{mg} / \mathrm{l}$ deste elemento) coincidem com os relatados por ZIEGLER (1990), que constatou teores entre $3,0 \mu \mathrm{g} / \mathrm{l}$ e $90 \mu \mathrm{g} / \mathrm{l}$.

\section{CONCLUSÕES}

Os teores de cádmio diminuem durante o transcorrer das fermentações e após a refrigeração dos vinhos.Os níveis de cádmio encontrados no produto final (vinho) estão dentro dos valores considerados normais pela O.I.V., à exceção do vinho oriundo do mosto adicionado com $5 \mathrm{mg} / \mathrm{l}$ deste elemento.

\section{AGRADECIMENTOS}

Os autores agradecem ao $\mathrm{CNPq}$ pelo financiamento parcial do trabalho e à Almadén Vinhos Finos Ltda. pela colaboração.

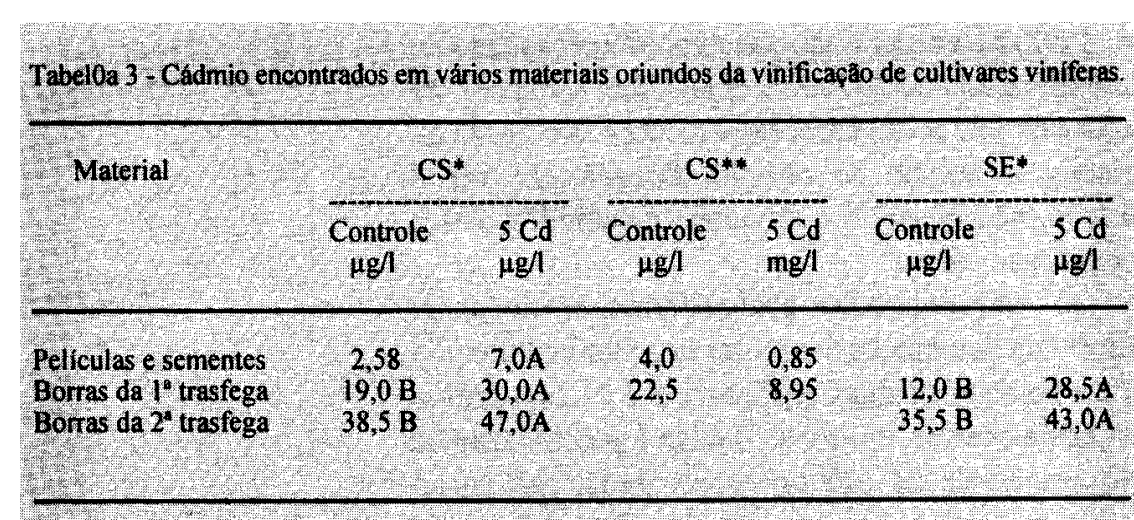

Na horizontal, medias com letras iguais nalo díerem pelo teste de Duncan a $5 \%$ de significancia - CS e SE = Cahernet Sauvignon e Saint Emilion provenientes de Santana do Livramento. * $\mathrm{CS}=$ Cabenet Sauvignon proveniente de Sto Sepé.

Ciência Rural, v. 27, n. 1, 1997. 


\section{REFERENCIAS BIBLIOGRÁFICAS}

AMERINE, M.A., OUGH, C.S. Wine and must analysis. New York: J. Wiley \& Sons, 1974, 121 p.

ANDREY, D., BEUGGERT, H., CESCHI, M., et al Monitoring program for heavy metais in food. Part. 4. Lead, cadmium, copper and zinc in wine ofthe Swiss market. Part B. Methods, results and discussion. Mitt. Geb. Lebensmittelunters. Hyg., v. 83, n. 6,p.711-736, 1992.

ANGLE, J.S, McGRATH, S.P., CHAUDRI, A.M. Effects of media components on toxicity of cadmium to rhizobia. Water, Air, Soil Pollut., v. 64, n. 3/4, p. 627-633, 1992.

ARCOS, M.T, ANCIN, M.C, ECHEVERRIA, J.C. et al Study of lability of heavy metais in wines with different degrees ofaging through difíerential pulse anodic stripping voltametry. J Agric Food Chem, v. 41, n. 12, p. 2333-2339, 1993.

COTTON, F.A., W1LK.INSON, G. Química Inorgânica. Rio de Janeiro: Livros Técnicos e Científicos, 1978. 600 p.

DAUDT, C.E, DAL PIVA, G.G. Determinação de elementos minerais em mostos e vinhos. In: CONGRESSO BRASILEIRO DE CIÊNCIA E TECNOLOGIA DE ALIMENTOS, Rio de Janeiro, 1989. Anais ...Rio de Janeiro: SBPC, 1989. p. 15-19.

DAUDT, C.E, DAL PIVA, G.G., RIZZON, L.A. Minerais em mostos e vinhos oriundos de uvas tratadas com fungicida Dimane M-45. Boletim da Sociedade Brasileira de Ciência e Tecnologia de Alimentos, Campinas, v. 26, n. 2, p. 81-88 1992.

DAUDT, C.E. GARCIA, N.G. Minerais em videiras, mostos e vinhos brasileiros. I. Minerais em vinhos. Ciência e Tecnologia de Alimentos, Campinas, v. 7, n. 1, p. 72-81, 1987.

DAUDT, C.E., GARCIA, N.G., RIZZON, L.A. Minerais em videiras, mostos e vinhos. II. Minerais em mostos, sua utilização durante a fermentação alcoólica e presença em vinhos. Ciência e Tecnologia de Alimentos, Campinas, v. 7 , n.2,p.189-204,1987.

DELAS, J. Lês toxicites metailiques dans lês sois acides. Prog Agric Vitic, Montpeilier, v. 101, n. 4, p. 96-101, 1984.

ENKELMANN, R. Effeets of cadmium sulfate in soils on cadmium uptake by grapevine (Viris vinifera L.). Angew Bot,

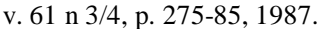

ESCHENBRUCH, R. Sulfite and sulfide formation during winemaking - a review. Am J Enol Vitic, v. 25, n 3 p 157 161,1974 .
ESCHNAUER, H. Trace elements in must and wine: primary and secondary contents. Am J Enol Vitic, v. 33, n. 4, p. 226-30, 1982.

GAERTEL, W. Heavy metais in pesticides as impurities: their importance as contaminants of wine. Nachrichtenbl. Dtsch. Pflanzenschutzdiensten. Braunschweig, v. 36, n. 10, p. 1547, 1984.

KOFOD, P., BAUER, R., DANIELSEN, E., et al. Cadmium -113 NMR investigation of a cadmium-substituted copper, zinccontaining superoxide dismutase from yeast. Eiir J Biochem, v. 198, n. 3, p. 607-611, 1991.

LEMEN, R.A., LEE, J.S., WAGONER, J.K. Mortality among workers exposed to cadmium. [S.P.:s.n.], 1975. (Presented at the New York Academy of Science meeting on Occupational Carcinogenesis, March, 1975).

MOHR, H.D. Studies on the location of heavy metais added to grape must, after fermentation. Weinberg Keller., v. 26, n. 7, p.277-288, 1979.

OLASUPO, N.A., SCOTT-EMNAKPOR. M.B., OGUNSHOLA, R.A. Resistance to heavy metais by some Nigerian yeast strains. Folia Microbiologica, [s.1.], v. 38. n. 4, p. 285-287, 1993.

ROSENEGGER, P. Environmental eontaminants in food. Ciênc Tecnol Aliment, Campinas, v. 12, n. 2, p. 197-198, 1992.

RUPP, D., RÜHL, E., ALLEWELDT, G. Cadmiumtoxizitat bei Weinreben. Vitis, v. 24, p. 88-96, 1985.

STEEL, R.G.D, TORR1E, J.H. Principies and Procedures of Statiscs. 2. ed.. New York: Mc Graw-Hill, 1980.610 p.

URENA-POZO, M.E., GIMÉNEZ-PLAZA, J., CANO-PAVÓN, J.M. Estúdio dei contenido de trazas de hierro, manganeso, cobre, piomo, cinc, cádmio y cobalto en los vinos de Málaga. Alimentaria, Madrid, p. 83-86, marzo 1987.

WENCKER, D., SPIESS, B., LAUGEL, P. Influence of hexacyanoferrate (II) based treatments upon the elimination of heavy metal traces in wine. II - The case of cadmium. Food additives and contaminants, v. 7, n. 3, p. 375-379, 1990.

WINGE, D.R., DAMERON. C.T., MEHRA, R.K. Metal: sulfide quantum crystailites in yeast. Detoxification of cadmium ions in yeast by formation ofcadmium-sulfide quantum crystailites in yeast: Biosynthesis and properties of ciystailites. In: Metallothioneins New York: VCH, 1992. p. 257-270.

ZIEGLER, B. Nutrients and heavy metal content. Wein-wiss, v. 45 n. 1, p, 24-26, 1990.

Ciência Rural, v. 27n. 11997 